\title{
Traumatisierungen bei Psychosepatienten: Weitere Argumente gegen das „bio-bio-bio Modell“?
}

\author{
Traumatic Experiences in Patients with Psychosis: More Arguments \\ Against the „bio-bio-bio Model“?
}

Autor

Institut
Ingo Schäfer

Klinik für Psychiatrie und Psychotherapie, Universitätsklinikum Hamburg-Eppendorf

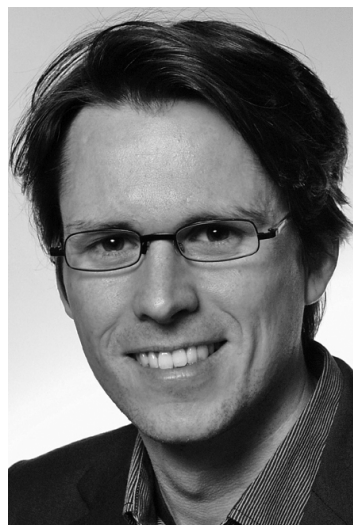

Dr. med. Ingo Schäfer
Bibliografie

DOI $10.1055 / \mathrm{s}-0029-1220374$

Psychiat Prax 2009; 36:

205-207

(c) Georg Thieme Verlag KG Stuttgart · New York .

ISSN 0303-4259

Korrespondenzadresse Dr. med. Ingo Schäfer, MPH Klinik für Psychiatrie und Psychotherapie, Universitätsklinikum Hamburg-Eppendorf Martinistraße 52 20246 Hamburg i.schaefer@uke.uni-hamburg.de
Von Steven Sharfstein, 2005-2006 Präsident der „American Psychiatric Association“, stammen einige pointierte Stellungnahmen. So müsse sich die Psychiatrie als Disziplin fragen, inwieweit sie zugelassen habe, dass „aus dem bio-psycho-sozialen Modell ein bio-bio-bio Modell“" geworden sei [1]. Ähnlich provokant erschien auf den ersten Blick Sharfsteins Aussage, Gewalt in der Kindheit habe „denselben Stellenwert für die Psychiatrie wie Zigarettenrauchen für die restliche Medizin“ [2]. Vor dem Hintergrund von Studien aus den letzten zwei Jahrzehnten, die den Stellenwert früher Traumatisierungen als bedeutsamen Risikofaktor für spätere psychische Störungen belegen, handelt es sich dabei eher um eine nüchterne Feststellung. Sowohl prospektive populationsbasierte Studien als auch Zwillingsstudien fanden nach Kontrolle weiterer psychosozialer Risikofaktoren, dass alle darin untersuchten psychischen Störungen nach früher Gewalt häufiger auftraten, teilweise im Sinne einer Dosis-Wirkungs-Beziehung. Unter anderem betraf dies Angststörungen, affektive Störungen, Essstörungen, Persönlichkeitsstörungen und Suchterkrankungen [3-5]. Im Gegensatz dazu waren zum Zusammenhang zwischen frühen Traumatisierungen und psychotischen Erkrankungen lange keine methodisch anspruchsvollen Studien verfügbar.

In den letzten Jahren begann sich dies zu ändern. So wurden inzwischen zahlreiche populationsbasierte Studien publiziert, die Zusammenhänge zwischen traumatischen Erfahrungen in frühen Lebensphasen, zumeist sexueller und körperlicher Gewalt, und späteren psychotischen Syndromen nahe legen (Übersicht bei [6]). Exemplarisch sei die Untersuchung von Janssen et al. [7] genannt. Darin wurde eine repräsentative Bevölkerungsstichprobe von über 4000 Personen prospektiv im Hinblick auf das Auftreten psychotischer Symptome untersucht. Psychiatrisch unauffällige Personen, die sexuellen Missbrauch oder körperliche Misshandlung in der Kindheit berichteten, entwickelten in dieser Studie im Verlauf von 2 Jahren 7-fach wahrscheinlicher behandlungsbedürftige psychotische Symptome. Dies war auch nach Kontrolle möglicher weiterer Einflussvariablen, wie psychotischen Symptomen in der Familie, Drogenkonsum, Alter und Bildungsstand der Fall.

Mögliche Mechanismen, die zu einer Erhöhung des Psychoserisikos im Sinne einer „erworbenen Vulnerabilität“ oder „Sensitivierung“ [8] durch frühe Traumatisierungen führen, werden zum einen in den neurobiologischen Folgen traumatischer Erfahrungen vermutet. Dies betrifft etwa Veränderungen verschiedener Neurotransmittersysteme [9]. So ist gut belegt, dass frühe Traumatisierungen zu anhaltenden Veränderungen der neuroendokrinen Stressreaktivität führen, die auch für die Entstehung psychotischer Erkrankungen von Bedeutung sind $[10,11]$. Auch direkte Auswirkungen auf das dopaminerge System werden aufgrund von tierexperimentellen Befunden und ersten Humanstudien diskutiert [12]. Zum anderen könnten die kognitiven und emotionalen Langzeitfolgen früher Traumatisierungen zur späteren Entstehung psychotischer Erkrankungen beitragen [13]. So stehen negative emotionale Zustände, geringer Selbstwert und ungünstige kognitive Schemata, die häufig in der Folge früher Traumatisierungen auftreten, im Mittelpunkt neuerer psychologischer Modelle der Psychoseentstehung $[14,15]$.

Während Zusammenhänge zwischen frühen Traumatisierungen und Psychosen in den letzten Jahren zunehmend empirische Unterstützung finden, ist die Häufigkeit und klinische Bedeutung traumatischer Erfahrungen bei Psychosepatienten bereits seit Langem gut belegt. In einer neueren Übersicht fassten Morgan und Fisher [16] 20 Studien zu früher sexueller und körperlicher Gewalt bei dieser Patientengruppe zusammen. Sexueller Missbrauch wurde im Mittel von 
$42 \%$ der weiblichen und $28 \%$ der männlichen Patienten berichtet, körperliche Misshandlung von 35 bzw. 38\%. Mindestens eine der beiden Formen früher Gewalt berichteten unabhängig vom Geschlecht 50\% der Patientinnen und Patienten. In verschiedenen Studien wiesen betroffene Patienten ein jüngeres Erkrankungsalter auf und profitierten schlechter von rehabilitativen Maßnahmen [17,18]. Sie zeigten mehr depressive Symptome und Ängstlichkeit [18,19], mehr Suizidalität und selbstverletzendes Verhalten [17] und mehr Probleme in nahen Beziehungen [20]. Einer der am häufigsten berichteten Unterschiede betrifft ein signifikant stärkeres Ausmaß von Positivsymptomen bei traumatisierten Patienten, insbesondere von Halluzinationen verschiedener Sinnesmodalitäten [18,21]. Wiederholt wurde auch berichtet, dass die Inhalte von Wahn und Halluzinationen mit traumatischen Erfahrungen zusammenhingen. So wurden nach sexuellem Missbrauch mehr sexuell getönte Wahninhalte berichtet oder es handelte sich bei imperativen, zu Suizid oder selbstverletzendem Verhalten auffordernden Stimmen, um die der Täter $[22,23]$. Andere Untersuchungen fanden subtilere Zusammenhänge. Sie weisen darauf hin, dass Patientinnen und Patienten mit frühen Traumatisierungen akustische Halluzinationen häufiger als feindselig und bedrohlich erleben [24], oder diese mit Schuld- oder Schamgefühlen verbunden sind [25]. Schließlich werden Symptome der Posttraumatischen Belastungsstörung (PTBS), die zu einem schweren Verlauf und einer schlechteren Prognose beitragen, bei Patienten mit Psychosen häufig unter- bzw. fehldiagnostiziert [26,27]. Eine weitere, bei Psychosepatienten erst wenig untersuchte Folge früher traumatischer Erlebnisse, stellen dissoziative Symptome und deren Zusammenhänge mit psychotischem Erleben dar [28,29].

Aus den genannten Befunden ergeben sich verschiedene klinische Implikationen. So wurde in den letzten Jahren verstärkt gefordert, psychosoziale Behandlungsangebote „traumasensibler“ zu gestalten und unabhängig von ihrem jeweiligen Versorgungsauftrag wesentliche Grundprinzipien des Umganges mit traumatisierten Personen zu berücksichtigen [30,31]. Dies beinhaltet unter anderem die eigenen Strukturen und Arbeitsabläufe daraufhin zu überprüfen, wie viel Sicherheit und Autonomie sie vermitteln und die Gefahr potenzieller Retraumatisierungen konsequent zu minimieren. Ein weiteres wichtiges Element traumasensibler Behandlung stellt die systematische Diagnostik traumatischer Erfahrungen dar [32]. Weiter wurde in den letzten Jahren begonnen auch „traumaspezifische“ Interventionen an die Bedürfnisse von Menschen mit Psychosen und anderen schweren psychischen Erkrankungen anzupassen. Dazu zählen Therapieprogramme, die einen Schwerpunkt auf Stabilisierung und den Aufbau von Bewältigungsstrategien setzen [33,34]. Aber auch mit expositionsbasierten Interventionen liegen im Einzel- [35] und Gruppenformat [36,37] inzwischen positive Erfahrungen vor. Schließlich stellt die Arbeit mit „dialogfähigen“ Stimmen gerade bei traumatisierten Psychosepatienten einen vielversprechenden Therapieansatz dar [38].

Die ätiologische und klinische Bedeutung von Traumatisierungen für einen Teil der Patienten mit psychotischen Erkrankungen wird durch eine wachsende Anzahl empirischer Befunde gestützt. Vor diesem Hintergrund erscheint es wichtig, ihren Beitrag zur Entstehung und Aufrechterhaltung belastender Symptome in klinisch-therapeutische Überlegungen einzubeziehen. Schließlich lässt die wachsende Literatur zur Bedeutung negativer Entwicklungseinflüssen für die Entstehung von Psychosen aber auch Ansätze zur Primärprävention dieser Erkrankungen deutlich werden. Oder, um mit Steven Sharfstein zu sprechen:
„Interpersonelle Gewalt, besonders Gewalterlebnisse im Kindesalter, stellen die wichtigste einzelne Ursache psychischer Erkrankung dar, die vermeidbar wäre“" [2].

\section{Literatur}

1 Sharfstein S. From the President. Big Pharma and American Psychiatry: The Good, the Bad, and the Ugly. Psychiatric News 2005; 40 (16): 3

2 Sharfstein S. From the President. New Task Force Will Address Early Childhood Violence. Psychiatric News 2006; 41 (3): 3

3 Fergusson DM, Horwood JL, Lynskey MT. Childhood sexual abuse and psychiatric disoder in young adulthood: II. Psychiatric outcomes of childhood sexual abuse. J Am Acad Child Adolesc Psychiatry 1996; 35: 1365-1374

4 Kendler KS, Bulik CM et al. Childhood sexual abuse and adult psychiatric and substance use disorders in women: an epidemiological and cotwin control analysis. Arch Gen Psychiatry 2000; 57: 953-959

5 Nelson EC, Heath AC et al. Association between self-reported childhood sexual abuse and adverse psychosocial outcomes: results from a twin study. Arch Gen Psychiatry 2002; 59: 139-145

6 Schäfer I, Ross C, Read J. Childhood trauma in psychotic and dissociative disorders. In: Moskowitz A, Schäfer I, Dorahy M. Psychosis, Trauma and Dissociation: Emerging perspectives on severe psychopathology. London: John Wiley \& Sons, 2008: 137-144

7 Janssen I, Krabbendam L et al. Childhood abuse as a risk factor for psychotic experiences. Acta Psychiatr Scand 2004; 109 (1): 38-45

8 Collip D, Myin-Germeys I, van Os J. Does the concept of „sensitization“ provide a plausible mechanism for the putative link between the environment and schizophrenia? Schizophr Bull 2008; 34 (2): 220-225

9 Read J, Perry BD, Moskowitz A et al. The contribution of early traumatic events to schizophrenia in some patients : a traumagenic neurodevelopmental model. Psychiatry 2001; 64: 319-345

10 Walker EF, Diforio D. Schizophrenia: a neural diathesis-stress model. Psychol Rev 1997; 104: 667-685

11 Myin-Germeys I, van Os J. Stress-reactivity in psychosis: evidence for an affective pathway to psychosis. Clin Psychol Revi 2007; 27: 409424

12 Spauwen J, Krabbendam L, Lieb R et al. Impact of psychological trauma on the development of psychotic symptoms: relationship with psychosis proneness. Brit J Psychiatr 2006; 188: 527-533

13 Fowler $D$, Freeman $D$ et al. The catastrophic interaction hypothesis: how do stress, trauma, emotion and information processing abnormalities lead to psychosis? In: Larkin W, Morrison AP. Trauma and psychosis. New directions for theory and therapy. London: Routledge, 2006: 101-124

14 Smith B, Fowler DG et al. Emotion and psychosis: links between depression, self-esteem, negative schematic beliefs and delusions and hallucinations. Schizophr Res 2006; 86 (1-3): 181-188

15 Bentall RP, Rowse $G$ et al. The cognitive and affective structure of paranoid delusions. A transdiagnostic investigation of patients with schizophrenia spectrum disorders and depression. Arch Gen Psychiatry 2009; 66 (3): 236-247

16 Morgan C, Fisher H. Environment and schizophrenia: environmental factors in schizophrenia: childhood trauma - a critical review. Schizophr Bull 2007; 33 (1): 3-10

17 Darves-Bornoz JM, Lemperiere $T$ et al. Sexual victimization in women with schizophrenia and bipolar disorder. Soc Psychiatry Psychiatr Epidemiol 1995; 30: 78-84

18 Lysaker PH, Davis LW, Gatton MJ et al. Associations of anxiety-related symptoms with reported history of childhood sexual abuse in schizophrenia spectrum disorders. J Clin Psychiatry 2005; 66: 1279-1284

19 Scheller-Gilkey G, Thomas SM, Woolwine BJ et al. Increased early life stress and depressive symptoms in patients with comorbid substance abuse and schizophrenia. Schizophr Bull 2002; 28: 223-231

20 Lysaker PH, Wickett AM, Lancaster RS et al. Neurocognitive deficits and history of childhood abuse in schizophrenia spectrum disorders: associations with Cluster B personality traits. Schizophr Res 2004; 68: 87-94

21 Read J, Agar K, Argyle $N$ et al. Sexual and physical abuse during childhood and adulthood as predictors of hallucinations, delusions and thought disorder. Psychol Psychother 2003; 76: 1-22

22 Ross CA, Anderson G, Clark P. Childhood abuse and the positive symptoms of schizophrenia. Hospital and Community Psychiatry 1994; 45: 489-491 
23 Read J, Argyle N. Hallucinations, delusions and thought disorder among adult psychiatric inpatients with a history of child abuse. Psychiatric Services 1999; 50: 1467-1471

24 Offen L, Waller G, Thomas $G$. Is reported childhood sexual abuse associated with the psychopathological characteristics of patients who experience auditory hallucinations? Child Abuse Negl 2003; 27: 919-927

25 Hardy A, Fowler D et al. Trauma and Hallucinatory Experience in Psychosis. J Nerv Ment Dis 2005; 193: 501-507

26 Resnick SG, Bond GR, Mueser KT. Trauma and posttraumatic stress disorder in people with schizophrenia. J Abnorm Psychol 2003; 112: 415-423

27 Neria $Y$, Bromet E, Sievers $S$ et al. Trauma exposure and posttraumatic stress disorder in psychosis: findings form a first-admission cohort. J Consult Clin Psychol 2002; 70: 246-251

28 Schäfer I, Harfst T et al. Childhood trauma and dissociation in female patients with schizophrenia spectrum disorders: An exploratory study. J Nerv Ment Dis 2006; 194: 135-138

29 Schäfer I, Spitzer C, Aderhold V et al. Dissociative symptoms in Schizophrenia. In: Moskowitz A, Schäfer I, Dorahy M. Psychosis, Trauma and Dissociation: Emerging perspectives on severe psychopathology. London: John Wiley \& Sons, 2008: 151-160

30 Rosenberg $S$, Mueser $K$ et al. Developing effective treatments for posttraumatic stress disorder among people with severe mental illness. Psychiatric Services 2001; 52: 1453-1461
31 Schäfer I. Traumasensible Therapie bei psychosekranken Menschen. Die Kerbe - Forum für Sozialpsychiatrie 2009; 27: 35-38

32 Read J, Hammersley P, Rudegeair T. Why, when and how to ask about childhood abuse. Advances in Psychiatric Treatment 2007; 13: 101-110

33 Fallot RD, Harris M. The Trauma Recovery and Empowerment Model (TREM): conceptual and practical issues in a group intervention for women. Community Ment Health J 2002; 38: 475-485

34 Najavits LM. Posttraumatische Belastungsstörung und Substanzmissbrauch. Das Therapieprogramm „Sicherheit finden“. Dt. Übersetzung und Bearbeitung von I. Schäfer, M. Stubenvoll, A. Dilling. Göttingen: Hogrefe Verlag, 2009

35 Callcott PS, Standart P. Trauma within psychosis: using a CBT model for PTSD in psychosis. Behavioural and Cognitive Psychotherapy 2004; 32: 239-244

36 Mueser K, Rosenberg S et al. A Randomized Controlled Trial of Cognitive-Behavioral Treatment Posttraumatic Stress Disorder in Severe Mental Illness. J Consult Clin Psychology 2008; 76 (2): 259-271

37 Frueh $C B$, Grubaugh $A L$ et al. Exposure-based cognitive-behavioral treatment of PTSD in adults with schizophrenia or schizoaffective disorder: A pilot study. Journal of Anxiety Disorders 2009; 23 (5): 665675

38 Romme M, Escher S. Stimmenhören verstehen. Der Leitfaden zur Arbeit mit Stimmenhören. Bonn: Psychiatrie-Verlag, 2008 